\section{Tolerance to Quinclorac by Seedling Creeping Bentgrass}

\author{
Zachary J. Reicher ${ }^{\mathbf{1}}$ and Glenn A. Hardebeck \\ Department of Agronomy, Purdue University, West Lafayette, IN 47907-1150
}

Fred F. Yelverton

Crop Science Department, North Carolina State University, Raleigh, NC 27695-7620

Nick E. Christians and Barbara Bingaman

Department of Horticulture, Iowa State University, Ames, IA 50011-1100

Jay Turner

TopPro Specialties, Germantown, TN 38138

Additional index words. siduron, crabgrass, herbicide, preemergence, postemergence

Abstract. Annual grassy weeds often inhibit establishment of spring-seeded creeping bentgrass (Agrostis palustris Huds.) on golf courses. The objective of this experiment was to evaluate the safety of the annual grass herbicide quinclorac in spring-seeded creeping bentgrass in varying climatic regions of the United States. Experiments were initiated in Indiana, Iowa, and North Carolina in Spring 2000. Treatments included siduron at 6.72 $\mathrm{kg} \cdot \mathrm{ha}^{-1}$ a.i. applied immediately prior to planting (PRE), and quinclorac at $0.84 \mathrm{~kg} \cdot \mathrm{ha}^{-1} \mathrm{a}$.i. applied 7 days before seeding (DBS), PRE, and 14 or 28 days after emergence (DAE). Herbicides were applied to three creeping bentgrass cultivars at each location. Siduron reduced establishment of 'Providence', 'L93', and 'Putter' creeping bentgrass in Indiana. Quinclorac applied PRE, 14 DAE, and 28 DAE caused short-term phytotoxicity, primarily in 'Providence' in Indiana. Quinclorac applications did not significantly affect cover of 'Providence', 'L93', or 'Putter' in Indiana or 'L93', 'Pennlinks', or 'Penncross' in Iowa. All applications of quinclorac reduced cover of 'L93', 'Pennlinks', and 'Penncross' in North Carolina. Though quinclorac applications resulted in no long-term damage when applied to creeping bentgrass seedlings in Indiana or Iowa, results from North Carolina indicate that caution should be exercised when using quinclorac on seedlings of creeping bentgrass. Chemical names used: 3,7-dichloro-8-quinolinecarboxylic acid (quinclorac); 1-(2-methylcyclohexyl)-3-phenylurea (siduron).

Creeping bentgrass is often seeded on golf courses during spring and early summer in new construction projects or after winter damage of existing turf. However, crabgrass (Digitaria spp.) often outcompetes creeping bentgrass seeded at this time of year. Siduron is labeled for use in creeping bentgrass and can be applied at time of seeding to effectively control crabgrass with little risk of damage to seedlings (Moshier et al., 1976). However, siduron has short residual activity and repeat applications are often needed for season-long crabgrass control. Other preemergence or postemergence herbicides labeled for use in cool-season turf have restrictions limiting use in seedling turf, on creeping bentgrass, or both. Quinclorac was labeled in 1998 for turfgrass use and has both preemergence and postemergence activity on large crabgrass (Digitaria sanguinalis L.) (Enache and Ilnicki, 1991) and provides control of a variety of broadleaf weeds including white clover (Trifolium reptans L.) (Neal, 1990). Label recommendations allow application of

Received for publication 4 Oct. 2000. Accepted for publication 19 Mar. 2001. Purdue Univ. Agricultural Experiment Station Journal No. 16387.

${ }^{1}$ To whom requests for reprints should be addressed. E-mail: zreicher@purdue.edu quinclorac to cool-season turf immediately before seeding, but applications after seeding are restricted to $28 \mathrm{~d}$ after emergence (DAE).
Earlier work has shown that quinclorac is safe to use on Kentucky bluegrass (Poa pratensis L.) or perennial ryegrass (Lolium perenne L.) at emergence and within 14 DAE (Reicher et al., 1999). Since creeping bentgrass is planted on golf courses worldwide, it is important to evaluate the safety of quinclorac on newly seeded creeping bentgrass. The objective of this experiment was to evaluate the safety of quinclorac in spring-seeded creeping bentgrass in varying climatic regions of the United States.

\section{Materials and Methods}

Experiments were initiated in Spring 2000 at three sites, including Purdue Univ.'s William H. Daniel Turfgrass Research and Diagnostic Center in West Lafayette, Ind.; Turfgrass Field Laboratory at North Carolina State Univ. in Raleigh; and Iowa State Univ. Horticulture Research Station in Ames. Seedbeds were prepared at each site by controlling vegetation with glyphosate $\{N$-(phosphonomethyl) glycine \}, tilling to a depth of $15 \mathrm{~cm}$, smoothing, and applying starter fertilizer. Methyl bromide fumigation was also used following tilling in Indiana to limit weed competition. After seeding creeping bentgrass at $49 \mathrm{~kg} \cdot \mathrm{ha}^{-1}$, irrigation was applied as needed to encourage germination and establishment. Plots were mowed at $1.3 \mathrm{~cm}$ as needed with clippings returned. Specific details for each site are listed in Table 1.

A $3 \times 6$ factorial in a split plot design was used at each location with three cultivars of creeping bentgrass as main blocks and six herbicide treatments as subplots. Creeping bentgrass cultivars were selected for each location based upon their use in the respective state. 'L93', 'Putter', and 'Providence' were used in Indiana, while 'L93', 'Penncross', and 'Pennlinks' were used in Iowa and North Carolina. Herbicide treatments included an untreated plot (hereafter referred to as the check),
Table 1. Site information for experimental locations in Indiana, Iowa, and North Carolina.

\begin{tabular}{|c|c|c|c|}
\hline & Indiana & Iowa & North Carolina \\
\hline Seeding date & 12 May 2000 & 17 May 2000 & 17 Apr. 2000 \\
\hline Emergence date ${ }^{z}$ & 19 May 2000 & 29 May 2000 & 27 Apr. 2000 \\
\hline \multicolumn{4}{|l|}{ Application dates } \\
\hline $7 \mathrm{DBS}^{\mathrm{y}}$ & 3 May 2000 & 10 May 2000 & 10 Apr. 2000 \\
\hline $\mathrm{PRE}^{\mathrm{x}}$ & 12 May 2000 & 17 May 2000 & 17 Apr. 2000 \\
\hline $14 \mathrm{DAE}^{\mathrm{w}}$ & 2 June 2000 & 13 June 2000 & 11 May 2000 \\
\hline 28 DAE & 16 June 2000 & 27 June 2000 & 25 May 2000 \\
\hline \multirow[t]{5}{*}{ Soil type } & Silt loam & Loam & Sandy clay loam \\
\hline & $5.9 \mathrm{pH}$ & $7.4 \mathrm{pH}$ & $5.8 \mathrm{pH}$ \\
\hline & $\mathrm{P}$ at $>224 \mathrm{~kg} \cdot \mathrm{ha}^{-1}$ & $\mathrm{P}$ at $34 \mathrm{~kg} \cdot \mathrm{ha}^{-1}$ & $\mathrm{P}$ at $125 \mathrm{~kg} \cdot \mathrm{ha}^{-1}$ \\
\hline & $\mathrm{K}$ at $1043 \mathrm{~kg} \cdot \mathrm{ha}^{-1}$ & $\mathrm{~K}$ at $180 \mathrm{~kg} \cdot \mathrm{ha}^{-1}$ & $\mathrm{~K}$ at $53 \mathrm{~kg} \cdot \mathrm{ha}^{-1}$ \\
\hline & $7.3 \% \mathrm{OM}$ & $4.1 \% \mathrm{OM}$ & $1.8 \% \mathrm{OM}$ \\
\hline \multirow[t]{3}{*}{ Seedbed fertilizer } & $\mathrm{N}$ at $27 \mathrm{~kg} \cdot \mathrm{ha}^{-1}$ & $\mathrm{~N}$ at $49 \mathrm{~kg} \cdot \mathrm{ha}^{-1}$ & $\mathrm{~N}$ at $13 \mathrm{~kg} \cdot \mathrm{ha}^{-1}$ \\
\hline & $\mathrm{P}$ at $32 \mathrm{~kg} \cdot \mathrm{ha}^{-1}$ & $\mathrm{P}$ at $49 \mathrm{~kg} \cdot \mathrm{ha}^{-1}$ & $P$ at $1 \mathrm{~kg} \cdot \mathrm{ha}^{-1}$ \\
\hline & $\mathrm{K}$ at $44 \mathrm{~kg} \cdot \mathrm{ha}^{-1}$ & $\mathrm{~K}$ at $0 \mathrm{~kg} \cdot \mathrm{ha}^{-1}$ & $\mathrm{~K}$ at $1 \mathrm{~kg} \cdot \mathrm{ha}^{-1}$ \\
\hline \multicolumn{4}{|l|}{ Postseeding fertilizer } \\
\hline Application date & 42 DAE & None & 28 and 56 DAE \\
\hline \multirow{3}{*}{ Application rate } & $\mathrm{N}$ at $37 \mathrm{~kg} \cdot \mathrm{ha}^{-1}$ & None & $\mathrm{N}$ at $13 \mathrm{~kg} \cdot \mathrm{ha}^{-1}$ \\
\hline & $\mathrm{P}$ at $0 \mathrm{~kg} \cdot \mathrm{ha}^{-1}$ & --- & $\mathrm{P}$ at $1 \mathrm{~kg} \cdot \mathrm{ha}^{-1}$ \\
\hline & $\mathrm{K}$ at $0 \mathrm{~kg} \cdot \mathrm{ha}^{-1}$ & --- & $\mathrm{K}$ at $1 \mathrm{~kg} \cdot \mathrm{ha}^{-1}$ \\
\hline Replications of main plots & 3 & 3 & 4 \\
\hline Subplot size & $1.5 \times 1.5 \mathrm{~m}$ & $1.5 \times 1.5 \mathrm{~m}$ & $1.5 \times 1.2 \mathrm{~m}$ \\
\hline
\end{tabular}


siduron at $6.72 \mathrm{~kg} \cdot \mathrm{ha}^{-1}$ a.i. applied immediately before planting (PRE), and quinclorac at $0.84 \mathrm{~kg} \cdot \mathrm{ha}^{-1}$ a.i. applied $7 \mathrm{~d}$ before seeding (DBS), PRE, 14, or $28 \mathrm{~d}$ after emergence (DAE). Emergence was defined as a uniform stand of at least one-leaf seedlings on each untreated plot. Herbicides were applied with $\mathrm{CO}_{2}$ powered sprayers in $816 \mathrm{~L} \cdot \mathrm{ha}^{-1}$ water and watered-in with at least $0.6 \mathrm{~cm}$ water within 2 $\mathrm{d}$ of application.

Cover of creeping bentgrass was visually rated weekly as a percentage cover of each plot. Phytotoxicity was rated weekly on a scale of 1 to 9 where $1=$ brown turf, $5=$ acceptable damage and $9=$ no visible phytotoxicity. Visual quality was rated weekly on a scale of 1 to 9 where $1=$ dead turf, $5=$ acceptable and 9 $=$ excellent quality. Percentage of weed cover was rated weekly in the Iowa study. Data from each site were analyzed in split block designs with PROC ANOVA (SAS Institute, 198996). Data recorded before 35 DAE were analyzed as a generalized randomized block with multiple replications of the untreated check within each block. Fischer's protected LSD was calculated using appropriate error terms according to Steel and Torrie (1980) and were used to separate treatment means.

\section{Results and Discussion}

Indiana. Siduron applied PRE caused noticeable phytotoxicity at 7 DAE (Table 2), but phytotoxicity was minimal and lasted less than a week. Quinclorac applied 14 DAE caused phytotoxicity at 21 DAE, but again this phytotoxicity was short-lived and caused no permanent detrimental effects. However, quinclorac applied at $28 \mathrm{DAE}$ caused phytotoxicity lasting from 35 to 49 DAE. Phytotoxicity appeared as a yellowing of older leaf blades and tips. A significant cultivar $\times$ herbicide interaction indicated that 'Providence' was slightly more sensitive to quinclorac than either ' $\mathrm{L} 93$ ' or 'Putter'.

Siduron applied PRE reduced creeping bentgrass cover from 7 to 35 DAE in Indiana (Table 3). This is in contrast to a study that showed siduron to be safe on seedlings of 'Penncross' and 'Seaside' creeping bentgrass (Moshier et al., 1976). Any possible negative effects of siduron may be compensated for by improved annual grass control in the majority of uses, but fumigation at the Indiana site removed all weed competition. A significant cultivar $\times$ herbicide interaction revealed that siduron decreased cover of 'Putter' and 'L93' at 21 DAE, but it had no effect on cover of 'Providence'. 'Providence' seedlings were more aggressive, establishing faster than ' $L 93$ ' and 'Putter', and outgrowing any potential damaging effects of siduron. Negative effects of quinclorac on creeping bentgrass cover were minimal in Indiana. Only quinclorac applied PRE reduced bentgrass cover compared to the check at 35 DAE by $2 \%$.

Iowa. Unlike the Indiana study, there was no cultivar $\times$ herbicide interaction at any time during the study and means of herbicide treat-

Table 2. Phytotoxicity ratings on 'L93', 'Providence' (Prov), and 'Putter' creeping bentgrass treated with siduron or quinclorac either before or after seeding in Indiana.

\begin{tabular}{|c|c|c|c|c|c|c|c|c|c|c|c|c|c|c|c|c|c|}
\hline \multirow[b]{2}{*}{ Herbicide } & \multirow{2}{*}{$\begin{array}{l}\text { Application } \\
\text { timing }\end{array}$} & \multicolumn{4}{|c|}{$7 \mathrm{DAE}^{\mathrm{y}}$} & \multicolumn{4}{|c|}{$21 \mathrm{DAE}$} & \multicolumn{4}{|c|}{$35 \mathrm{DAE}$} & \multicolumn{4}{|c|}{$49 \mathrm{DAE}$} \\
\hline & & L93 & Prov & Putter & Mean & L93 & Prov & Putter & Mean & L93 & Prov & Putter & Mean & L93 & Prov & Putter & Mean \\
\hline Check & --- & $7.4^{x}$ & 7.3 & 7.6 & 7.4 & 9.0 & 9.0 & 9.0 & 9.0 & 9.0 & 8.7 & 9.0 & 8.9 & 9.0 & 9.0 & 9.0 & 9.0 \\
\hline Quinclorac & $7 \mathrm{DBS}^{\mathrm{w}}$ & 7.3 & 7.7 & 7.7 & 7.6 & 9.0 & 9.0 & 9.0 & 9.0 & 9.0 & 8.7 & 9.0 & 8.9 & 9.0 & 9.0 & 9.0 & 9.0 \\
\hline Siduron & $\mathrm{PRE}^{\mathrm{v}}$ & 7.0 & 6.0 & 6.7 & 6.6 & 9.0 & 9.0 & 9.0 & 9.0 & 9.0 & 8.7 & 9.0 & 8.9 & 9.0 & 9.0 & 9.0 & 9.0 \\
\hline Quinclorac & PRE & 7.0 & 7.0 & 7.3 & 7.1 & 9.0 & 9.0 & 9.0 & 9.0 & 9.0 & 8.7 & 9.0 & 8.9 & 9.0 & 9.0 & 9.0 & 9.0 \\
\hline Quinclorac & $14 \mathrm{DAE}$ & & & & & 7.7 & 5.3 & 6.7 & 6.6 & 9.0 & 8.7 & 9.0 & 8.9 & 9.0 & 9.0 & 9.0 & 9.0 \\
\hline Quinclorac & $28 \mathrm{DAE}$ & & & & & & & & & 7.3 & 4.7 & 5.0 & 5.7 & 8.3 & 7.3 & 8.3 & 8.0 \\
\hline \multicolumn{18}{|c|}{ Analysis of variance } \\
\hline Cultivar (C) & & \multicolumn{4}{|c|}{$*$} & \multicolumn{4}{|c|}{ NS } & \multicolumn{4}{|c|}{ NS } & \multirow{2}{*}{\multicolumn{4}{|c|}{$* *$}} \\
\hline Herbicide $(\mathrm{H})$ & & \multicolumn{4}{|c|}{$* *$} & \multicolumn{4}{|c|}{$* *$} & \multicolumn{4}{|c|}{$* *$} & & & & \\
\hline $\mathrm{C} \times \mathrm{H}$ & & \multicolumn{4}{|c|}{ NS } & \multicolumn{4}{|c|}{$* *$} & \multicolumn{4}{|c|}{$* *$} & \multicolumn{4}{|c|}{$* *$} \\
\hline $\mathrm{LSD}_{0.05}$ for comp & ing $\mathrm{C}$ means & \multicolumn{4}{|c|}{ NS } & \multicolumn{4}{|c|}{0.3} & \multicolumn{4}{|c|}{ NS } & \multicolumn{4}{|c|}{ NS } \\
\hline $\mathrm{LSD}_{0.05}$ for com & ing $\mathrm{H}$ means & \multicolumn{4}{|c|}{0.4} & \multicolumn{4}{|c|}{0.4} & \multicolumn{4}{|c|}{0.5} & \multicolumn{4}{|c|}{0.2} \\
\hline $\mathrm{LSD}_{0.05}$ for comp & ing $\mathrm{C} \times \mathrm{H}$ mea & & & NS & & & & 7 & & & & .9 & & \multicolumn{4}{|c|}{0.4} \\
\hline
\end{tabular}

${ }^{2}$ Phytotoxicity rated on a scale of 1 to 9 where $1=$ brown turf, $5=$ acceptable damage and $9=$ no visible phytotoxicity.

yDays after emergence.

${ }^{x}$ Means of 3 replications.

${ }^{w}$ Days before seeding.

vPreemergence application made immediately before planting.

Ns, *,**Nonsignificant or significant at $P=0.05$ or $P=0.01$, respectively.

Table 3. Percent cover of 'L93', 'Providence', and 'Putter' creeping bentgrass treated with siduron or quinclorac either before or after seeding in Indiana.

\begin{tabular}{|c|c|c|c|c|c|c|c|c|c|c|c|c|c|c|c|c|c|}
\hline \multirow[b]{2}{*}{ Herbicide } & \multirow{2}{*}{$\begin{array}{c}\text { Application } \\
\text { timing }\end{array}$} & \multicolumn{4}{|c|}{$7 \mathrm{DAE}^{\mathrm{z}}$} & \multicolumn{4}{|c|}{$21 \mathrm{DAE}$} & \multicolumn{4}{|c|}{$35 \mathrm{DAE}$} & \multicolumn{4}{|c|}{$49 \mathrm{DAE}$} \\
\hline & & L93 & Prov & Putter & Mean & L93 & Prov & Putter & Mean & L93 & Prov & Putter & Mean & L93 & Prov & Putter & Mean \\
\hline & & -- & -- & --- & --- & --- & -- & --- & --- & $\%-$ & --- & --- & --- & -- & -- & --- & $\overline{---}$ \\
\hline Check & --- & $18^{y}$ & 73 & 15 & 35 & 86 & 99 & 82 & 89 & 98 & 100 & 99 & 99 & 99 & 100 & 100 & 100 \\
\hline Quinclorac & $7 \mathrm{DBS}^{\mathrm{x}}$ & 22 & 77 & 20 & 39 & 86 & 98 & 90 & 91 & 99 & 100 & 98 & 99 & 100 & 100 & 98 & 99 \\
\hline Siduron & $\mathrm{PRE}^{\mathrm{w}}$ & 13 & 68 & 7 & 29 & 73 & 96 & 60 & 76 & 94 & 100 & 94 & 96 & 97 & 100 & 97 & 98 \\
\hline Quinclorac & PRE & 20 & 67 & 20 & 36 & 83 & 97 & 78 & 86 & 96 & 99 & 97 & 97 & 98 & 99 & 97 & 98 \\
\hline Quinclorac & $14 \mathrm{DAE}$ & & & & & 82 & 99 & 83 & 88 & 96 & 100 & 98 & 98 & 98 & 100 & 99 & 99 \\
\hline Quinclorac & $28 \mathrm{DAE}$ & & & & & & & & & 99 & 100 & 97 & 98 & 100 & 99 & 98 & 99 \\
\hline \multicolumn{2}{|l|}{ Cultivar mean } & 18 & 71 & 15 & & 82 & 98 & 79 & & 97 & 100 & 97 & & 99 & 100 & 98 & \\
\hline \multicolumn{18}{|c|}{ Analysis of variance } \\
\hline Cultivar (C) & & \multicolumn{4}{|c|}{$* *$} & \multicolumn{4}{|c|}{ NS } & \multicolumn{4}{|c|}{ NS } & \multicolumn{4}{|c|}{ NS } \\
\hline Herbicide $(\mathrm{H})$ & & \multicolumn{4}{|c|}{$* *$} & \multicolumn{4}{|c|}{$* *$} & \multicolumn{4}{|c|}{$*$} & \multicolumn{4}{|c|}{ NS } \\
\hline $\mathrm{C} \times \mathrm{H}$ & & \multicolumn{4}{|c|}{ NS } & \multicolumn{4}{|c|}{$* *$} & \multicolumn{4}{|c|}{ NS } & \multicolumn{4}{|c|}{ NS } \\
\hline $\operatorname{LSD}_{0.05}$ for comp & g C means & \multicolumn{4}{|c|}{4} & \multicolumn{4}{|c|}{ NS } & \multicolumn{4}{|c|}{ NS } & \multicolumn{4}{|c|}{ NS } \\
\hline $\mathrm{LSD}_{0.05}$ for comp & g H means & \multicolumn{4}{|c|}{5} & \multicolumn{4}{|c|}{5} & \multicolumn{4}{|c|}{2} & \multicolumn{4}{|c|}{ NS } \\
\hline $\mathrm{LSD}_{0.05}$ for comp & $\mathrm{g} \mathrm{C} \times \mathrm{H}$ means & \multicolumn{4}{|c|}{ NS } & \multicolumn{4}{|c|}{8} & \multicolumn{4}{|c|}{ NS } & \multicolumn{4}{|c|}{ NS } \\
\hline
\end{tabular}

${ }^{2}$ Days after emergence.

${ }^{y}$ Means of 3 replications.

${ }^{x}$ Days before seeding.

wPreemergence application made immediately before planting.

Ns, ${ }^{* * * *}$ Nonsignificant or significant at $P=0.05$ or $P=0.01$, respectively. 
ments over all three cultivars are presented. None of the herbicide treatments reduced bentgrass cover compared to the check (Table 4). However, siduron-treated plots had greater bentgrass cover compared with the check and quinclorac applied PRE, 14 DAE, and 28 DAE when rated at 35 DAE (Table 4). This was primarily due to siduron controlling purslane (Portulaca oleracea L.) and prostrate pigweed (Amaranthus blitoides S.). Siduron reduced cover of these weed species by up to $21 \%$ compared with the check at 49 DEA, whereas quinclorac treatments did not reduce weed cover. Others have found quinclorac applied preemergence and postemergence to be effective on broadleaf weeds such as dandelion (Taraxacum officianale Weber) (Enache and Ilnicki, 1991) and effective on white clover (Trifolium repens $\mathrm{L}$.) when applied postemergence (Neal, 1990). Quinclorac had little effect on purslane and prostrate pigweed in IA either preemergence or postemergence. Quinclorac applied 7 DBS, PRE, and 14 DAE provided slightly better weed control than quinclorac applied 28 DAE. Similar to the Indiana study, quinclorac applied 14 DAE caused slight

Table 4. Percent cover of creeping bentgrass and weeds treated with siduron or quinclorac either before or after seeding in Iowa.

\begin{tabular}{|c|c|c|c|c|c|c|c|c|}
\hline \multirow[b]{2}{*}{ Herbicide } & \multirow{2}{*}{$\begin{array}{l}\text { Application } \\
\text { timing }\end{array}$} & \multicolumn{4}{|c|}{ Creeping bentgrass cover } & \multicolumn{3}{|c|}{ Weed cover ${ }^{\mathrm{a}}$} \\
\hline & & $7 \mathrm{DAE}^{\mathrm{z}}$ & $21 \mathrm{DAE}$ & $35 \mathrm{DAE}$ & $49 \mathrm{DAE}$ & $21 \mathrm{DAE}$ & $35 \mathrm{DAE}$ & $49 \mathrm{DAE}$ \\
\hline & & ---1 & ---9 & $0--$ & ---- & ---- & $\% \quad$ & ---- \\
\hline Check & --- & $25^{y}$ & 43 & 62 & 75 & 5 & 13 & 28 \\
\hline Quinclorac & $7 \mathrm{DBS}^{\mathrm{x}}$ & 26 & 47 & 67 & 74 & 4 & 13 & 21 \\
\hline Siduron & $\mathrm{PRE}^{\mathrm{w}}$ & 24 & 39 & 69 & 78 & 1 & 3 & 7 \\
\hline Quinclorac & PRE & 25 & 43 & 60 & 74 & 6 & 14 & 21 \\
\hline Quinclorac & $14 \mathrm{DAE}$ & 46 & 59 & 69 & 4 & 13 & 21 & \\
\hline Quinclorac & $28 \mathrm{DAE}$ & 58 & 67 & 19 & 34 & & & \\
\hline \multicolumn{9}{|c|}{ Analysis of variance } \\
\hline Cultivar (C) & & NS & NS & NS & NS & NS & NS & NS \\
\hline Herbicide $(\mathrm{H})$ & & NS & NS & $*$ & NS & $*$ & $*$ & $*$ \\
\hline $\mathrm{C} \times \mathrm{H}$ & & NS & NS & NS & NS & NS & NS & NS \\
\hline $\mathrm{LSD}_{0.05}$ for com & ring $\mathrm{H}$ means & NS & NS & 6 & NS & 3 & 6 & 9 \\
\hline
\end{tabular}

${ }^{2}$ Days after emergence.

y Means over 3 replications and 3 cultivars of creeping bentgrass ('L93', 'Penncross,' and 'Pennlinks').

${ }^{x}$ Days before seeding.

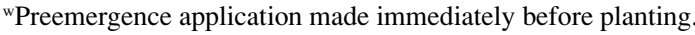

Ns, ${ }^{*}, * *$ Nonsignificant or significant at $P=0.05$ or $P=0.01$, respectively. ing Indiana (Reicher et al., 1999); western Virginia (Chism and Bingham, 1991); and New Jersey (Enache and Ilnicki, 1991). However, Georgia studies report quinclorac to be damaging to cool-season turf when exposed to heat stress (Johnson, 1994a). Further Georgia studies show quinclorac to be safe to creeping bentgrass when applied in February, but was damaging when applied in June (Johnson, 1994b). This could explain the marked differences in quinclorac effects in North Carolina, when compared to Indiana and Iowa. Air temperatures between 0 and $49 \mathrm{DAE}$ in Indiana never exceeded $32{ }^{\circ} \mathrm{C}$ and rarely exceeded 29 ${ }^{\circ} \mathrm{C}$, while air temperatures in Iowa exceeded $32{ }^{\circ} \mathrm{C}$ on only $4 \mathrm{~d}$ during this period. Air temperature in North Carolina exceeded $32^{\circ} \mathrm{C}$ on $22 \mathrm{~d}$ between 0 and 49 DAE. However, this may not explain the immediate reduction in creeping bentgrass cover caused by the 7 DBS and PRE applications of quinclorac in North Carolina. Evans et al. (1992) reported that quinclorac can be more damaging to coolseason turfgrasses when applied to soils with low organic matter. This may help to explain the damage caused by quinclorac applied 7 DBS and PRE in North Carolina. Organic matter content in the soils of Indiana and Iowa was $7.3 \%$ and $4.1 \%$, respectively, whereas organic matter content of the North Carolina soil was $1.8 \%$.

Quinclorac effects were fairly consistent among cultivars within each location, with only 'Providence' being slightly more sensitive to quinclorac resulting in phytotoxicity in Indiana. This agrees with Tapp and Powell (1992) who reported that quinclorac caused no injury when applied to 18 cultivars of 5 yearold creeping bentgrass.

Quinclorac applications resulted in no longterm damage when applied to 'L93', 'Pennlinks', and 'Penncross' in Iowa and to 'L93', 'Putter', and 'Providence' in Indiana. However, results from North Carolina where quinclorac damaged creeping bentgrass seedlings indicate that caution should be exercised when using quinclorac on seedlings of creeping bentgrass.

Table 5. Percent cover and visual quality of creeping bentgrass with siduron or quinclorac before or after seeding in North Carolina.

\begin{tabular}{|c|c|c|c|c|c|c|c|c|c|}
\hline \multirow[b]{2}{*}{ Herbicide } & \multirow{2}{*}{$\begin{array}{c}\text { Application } \\
\text { timing }\end{array}$} & \multicolumn{4}{|c|}{ Percent cover } & \multicolumn{4}{|c|}{ Visual quality $^{z}$} \\
\hline & & $21 \mathrm{DAE}^{\mathrm{y}}$ & $36 \mathrm{DAE}$ & $50 \mathrm{DAE}$ & $64 \mathrm{DAE}$ & $21 \mathrm{DAE}$ & $36 \mathrm{DAE}$ & $50 \mathrm{DAE}$ & $64 \mathrm{DAE}$ \\
\hline & & ---- & ---1 & --- & ---- & & & & \\
\hline Check & --- & $43^{\mathrm{x}}$ & 76 & 83 & 91 & 6.9 & 6.9 & 6.9 & 7.0 \\
\hline Quinclorac & $7 \mathrm{DBS}^{\mathrm{w}}$ & 31 & 50 & 61 & 69 & 5.8 & 5.8 & 5.7 & 5.7 \\
\hline Siduron & $\mathrm{PRE}^{\mathrm{v}}$ & 44 & 86 & 88 & 93 & 7.0 & 6.9 & 6.7 & 6.9 \\
\hline Quinclorac & PRE & 18 & 28 & 40 & 45 & 5.0 & 4.5 & 4.4 & 4.3 \\
\hline Quinclorac & $14 \mathrm{DAE}$ & 44 & 53 & 59 & 64 & 5.8 & 4.4 & 4.5 & 4.6 \\
\hline \multicolumn{10}{|c|}{ Analysis of variance } \\
\hline Cultivar (C ) & & NS & NS & NS & NS & NS & NS & NS & NS \\
\hline Herbicide $(\mathrm{H})$ & & $* *$ & $* *$ & $* *$ & $* *$ & $* *$ & $* *$ & $* *$ & $* *$ \\
\hline $\mathrm{C} \times \mathrm{H}$ & & NS & NS & NS & NS & NS & NS & NS & NS \\
\hline $\mathrm{LSD}_{0.05}$ for com & ng $\mathrm{H}$ means & 8 & 12 & 11 & 11 & 0.6 & 0.8 & 0.7 & 0.5 \\
\hline
\end{tabular}

${ }^{2}$ Visual quality rated on a scale of 1 to 9 where $1=$ dead turf, $5=$ acceptable and $9=$ excellent turf quality.

y Days after emergence.

${ }^{x}$ Means over 4 replications and 3 cultivars of creeping bentgrass ('L93', 'Penncross,' and 'Pennlinks').

wDays before seeding.

"Preemergence application made immediately before planting.

Ns, *, ${ }^{* *}$ Nonsignificant or significant at $P=0.05$ or $P=0.01$, respectively. 


\section{Literature Cited}

Chism, W.J. and S.W. Bingham. 1991. Postemergence control of large crabgrass (Digitaria sanguinalis) with herbicides. Weed Sci. 39:6266.

Enache, A.D. and R.D. Ilnicki. 1991. BAS 514 and dithiopyr for weed control in cool season turfgrasses. Weed Technol. 5:616-621.

Evans, J.R., W.J. Sciarappa, and A.L. Harrell. 1992. Quinclorac for weed control during turfgrass establishment. Southern Weed Sci. Soc. 45:134

Johnson, B.J. 1994a. Influence of dates and fre- quency of Drive treatments on large crabgrass control in tall fescue turf. J. Environ. Hort. 12(2):83-86.

Johnson, B.J. 1994b. Creeping bentgrass quality following preemergence and postemergence herbicide applications. HortScience 29:880-883.

Moshier, L., A.J. Turgeon, and D. Penner. 1976. Effects of glyphosate and siduron on turfgrass establishment. Weed Sci. 24(5):445-448.

Neal, J.C. 1990. Non-phenoxy herbicides for perennial broadleaf weed control in cool-season turf Weed Technol. 4:555-559.

Reicher, Z.J., D.V. Weisenberger, and C.S.
Throssell. 1999. Turf safety and effectiveness of dithiopyr and quinclorac for large crabgrass control in spring-seeded turf. Weed Technol. 13:253-256.

SAS Institute. 1989-96. Software Release 6.12. SAS Inst., Cary, N.C.

Steel, R.G. and J.H. Torrie. 1980. Principles and procedures of statistics: A biometrical approach. $2^{\text {nd }}$ ed. McGraw-Hill, New York.

Tapp, L.D. and A.J. Powell, Jr. 1992. Bentgrass tolerance to certain herbicides, p. 64. Kentucky Turfgrass Research 1990-92. Progress Rpt. 343. 